\title{
Symétrie et forme normale des centres et foyers dégénérés
}

\author{
ROBERT MOUSSU \\ Département de Mathématiques, Laboratoire de Topologie, Université de Dijon, \\ 21004 Dijon Cedex
}

(Received 18 January 1982)

Abstract. Consider an analytic differential equation $\omega=a d x+b d y$ with an algebraically isolated singularity and without a separatrix. The germ at $0 \in \mathbb{R}^{2}$ of the 1 -jet $y d y$ is either a focus or a centre. The equation has $C^{\infty}$ normal form of the type $\frac{1}{2} d\left(n y^{2}+x^{2 n}\right)+F(x) d y$ with $F(x)=F(-x)$ if and only if the germ is a centre.

\section{Introduction}

Dans cet article $\omega=a d x+b d y=0$ désigne le germe à l'origine $0 \in \mathbb{R}^{2}$ d'une équation différentielle $C^{\infty}$ (resp. analytique), à singularité algébriquement isolée; c'est à dire $a, b \in \mathscr{E}_{2}$ (resp. $O_{2}$ ) l'anneau des germes en $0 \in \mathbb{R}^{2}$ de fonctions $C^{\infty}$ (resp. analytiques) et $\operatorname{dim}_{\mathbf{R}} \mathscr{E}_{2} /\{a, b\}<\infty$.

Une séparatrice de $\omega$ est un germe en 0 de courbe $\gamma, C^{\infty}$, solution de $\omega=0$, qui n'est pas plat; c'est à dire $\gamma^{*}(\omega)=0$ et il existe $k>0$ tel que $\gamma^{(k)}(0) \neq 0$.

Lorsque l'équation $\omega=0$ n'a pas de séparatrice, il est bien connu depuis les travaux de Bendixson-Dulac-Poincaré, qu'à un germe de plongement de $(\mathbb{R}, 0)$ dans $\left(\mathbb{R}^{2}, 0\right)$, transverse à $\omega=0$, i.e $\tau^{*}(\omega)(t) \neq 0$ si $t \neq 0$, correspond un germe d'homéomorphisme $\boldsymbol{P}_{\tau}$ de $\left(\mathbb{R}^{+}, 0\right)$ appelé application premier retour de Poincaré. L'équation $\omega=0$ est dite: de type centre si $P_{\tau}=\left(1_{R^{+}}, 0\right)$, de type foyer si $P(t) \neq t$ pour $t \neq 0$, de type mixte si $P_{\tau}$ possède une infinité de points fixes.

L'équation $\omega=0$ est non dégénérée si l'une au moins des valeurs propres $\lambda_{i}$, $i=1,2$ de la matrice jacobienne

$$
M(\omega)=\frac{D(b,-a)}{D(x, y)}(0)
$$

est non nulle. Dans ce cas, $\omega=0$ ne possède pas de séparatrice si et seulement si $\lambda_{1}=\overline{\lambda_{2}} \notin \mathbb{R}$ et on a les résultats classiques:

* Si $\lambda_{1}+\lambda_{2} \neq 0, \omega=0$ est linéarisable, de type foyer [8].

** Si $\lambda_{1}+\lambda_{2}=0, \omega=0$ est de type centre si et seulement si elle s'écrit dans de bonnes coordonnées $d\left(x^{2}+y^{2}\right)=0[8],[6]$ sinon elle possède une forme normale [11]. 
L'équation $\omega=0$ est dégénérée de degré de dégénérescence 1 [3] si les deux valeurs propres de $M(\omega)$ sont nulles et $M(\omega) \neq 0$; c'est à dire le jet d'ordre 1 de $\omega$ en 0 s'écrit $\omega_{1}=y d y$ dans de bonnes coordonnées. Nous supposerons cette condition réalisée dans toute la suite. Le but de ce travail est de montrer comment se généralise alors * et **. Rappelons tout d'abord (avec nos notations) la proposition de 'mise' sous forme normale de Takens [12, p. 57].

Proposition 1. Il existe un $C^{\infty}$-difféomorphisme de $\left(\mathbb{R}^{2}, 0\right)$ tel que pour $q \in \mathbb{N} \cup \infty$, assez grand $(q \geq l, m)$, le jet d'ordre $q$ de $\varphi^{*}(\omega)$ en 0 s'écrive:

$$
\mathscr{T}^{q} \varphi^{*}(\omega)=\omega_{q}=\frac{1}{2} d\left(\frac{l}{2} y^{2}+\varepsilon x^{\prime}\right)+\left(\sum_{k=m}^{q} F_{k} x^{k}\right) d y
$$

où $l \geq 3, \varepsilon= \pm, m \geq 2$ et $F_{m} \neq 0$.

Dans [4], Lyapunov étudie une telle équation dans le cadre analytique. Certains de ses résultats qui restent vrais dans le cadre $C^{\infty}$ s'énoncent.

Proposition 2. L'équation $\omega=0$ ne possède pas de séparatrice si et seulement si:

$$
l=2 n, \quad \varepsilon=+, \quad m>n \quad \text { ou } m=n \text { et } F_{m}^{2}<4 m .
$$

Son application premier retour $P_{\tau}$, correspondant à un plongement $\tau$, est $C^{\infty}$ (analytique si $\omega$ est analytique).

Dans toute la suite $P\left(=P_{\tau}\right)$ désigne l'application premier retour de $\omega=0$ correspondant à un plongement $\tau$ tangent à l'axe $y=0$ et on appelle ordre de platitude de $\omega=0$, l'ordre $p(\omega) \in \mathbb{N} \cup \infty$ de $P(t)-t$ en 0 . Avec les notations de la proposition 1 on a:

Proposition 3. Si l'équation $\omega=0$ ne possède pas de séparatrice,

$$
p(\omega)=2 q(\omega)+2-n
$$

où $q(\omega)$ est le plus petit des q tels que $F_{2 q+1} \neq 0$; plus précisément il existe $C>0$ tel que

$$
P(t)-t=-C F_{2 q(\omega)+1} t^{p(\omega)}+\cdots .
$$

Ainsi $p(\omega)=\infty$ si et seulement si $\sum F_{k} x^{k}$ est pair. Plus précisément on a:

THÉORÈME 1. L'équation $\omega=0\left(\mathscr{T}^{1} \omega=y d y\right)$ est de type centre si et seulement si il existe un $C^{\infty}$-difféomorphisme $\varphi$ de $\left(\mathbb{R}^{2}, 0\right)$ tel que:

$$
\varphi^{*}(\omega)=\frac{1}{2} d\left(n y^{2}+x^{2 n}\right)+G\left(x^{2}\right) d y
$$

où $G\left(x^{2}\right)=x^{m} g\left(x^{2}\right), g(0) \neq 0$ et $n<m$ ou $n=m$ et $g(0)^{2}<4 m$.

Remarque 1. Contrairement à ce qui se passe pour une équation de type centre, non dégénérée, une équation $\omega=0$ vérifiant les hypothèses du théorème n'a pas en général d'intégrale première. En effet, supposons qu'il existe $h, H \in \mathscr{E}_{2}$ tels que

$$
h \omega=d H, \quad h(0) \neq 0 .
$$

Un résultat classique de Whitney permet d'écrire:

$$
\left(h_{1}\left(x^{2}, y\right)+x h_{2}\left(x^{2}, y\right)\right) \omega=d\left(H_{1}\left(x^{2}, y\right)+x H_{2}\left(x^{2}, y\right)\right)
$$

où $h_{1}, h_{2}, H_{1}, H_{2} \in \mathscr{C}_{2}$. De l'invariance de $\varphi^{*}(\omega)$ par l'involution

$$
(x, y) \rightarrow(-x, y),
$$


on déduit que

$$
h_{1}\left(x^{2}, y\right) \omega=d\left(H_{1}\left(x^{2}, y\right)\right), \quad h_{1}(0) \neq 0 .
$$

C'est à dire que $H_{1}(u, y)$ est une intégrale première de l'équation

$$
\alpha=\frac{1}{2} d\left(n y^{2}+u^{n}\right)+G(u) d y .
$$

Or, une telle équation n'a pas en 'général' d'intégrale première [9].

THÉoRèME 2. L'équation $\omega=0\left(\mathscr{T}^{-1} \omega=y d y\right)$ est de type foyer non plat $(p(\omega)<\infty)$ si et seulement si il existe un $C^{\infty}$-difféomorphisme $\varphi$ de $\left(\mathbb{R}^{2}, 0\right)$ tel que

$$
\varphi^{*}(\omega)=\frac{1}{2} d\left(n y^{2}+x^{2 n}\right)+F(x) d y
$$

où $\mathscr{T}^{\infty} F(x) \neq \mathscr{T}^{\infty} F(-x), F(x)=x^{m} f(x), f(0) \neq 0$ et $m<n$ ou $n=m$ et $f(0)^{2}<4 m$.

Remarque 2. Supposons $\omega$ analytique $\left(\mathscr{T}^{1} \omega=y d y\right)$. Alors $\omega=0$ est, d'après la proposition 2 , du type centre ou foyer non plat. Mais le difféomorphisme $\varphi$ des théorèmes 1 ou 2 est seulement $C^{\infty}$. Est-il possible de trouver un $\varphi$ analytique? Plus modestement si $\omega=0$ est du type centre existe-t-il une involution analytique $I$ telle que $I^{*}(\omega) \wedge \omega=0$ ?

Remarque 3. La proposition 2 et les théorèmes 1 et 2 s'appliquent aux équations différentielles du second ordre du type:

$$
\frac{d^{2} x}{d t^{2}}+f(x) \frac{d x}{d t}+x^{2 n-1}=0 .
$$

En effet cette équation s'écrit encore:

$$
\begin{aligned}
& \frac{d Y}{d t}=-Y f(x)-x^{2 n-1}, \\
& \frac{d x}{d t}=Y,
\end{aligned}
$$

ou, si l'on ne s'intéresse qu'aux orbites:

$$
\omega=Y d Y+\left(x^{2 n-1}+Y f(x)\right) d x=0 .
$$

En faisant le changement:

$$
y=Y+F(x) \quad \text { avec } F(0)=0, \quad f(x)=F^{\prime}(x),
$$

on obtient:

$$
\omega=y d y+x^{2 n-1} d x-F(x) d y .
$$

Si l'ordre de $f \in \mathscr{O}_{1}$ est supérieur à $n$, on déduit des résultats précédents que les solutions de (E) sont périodiques si et seulement si $f$ est impaire ou que, $0 \in \mathbb{R}$ est un attracteur ou un 'répulseur' si $f$ n'est pas impaire; le premier terme pair du développement de Taylor de $f$ permettant de décider si 0 est un attracteur ou un 'répulseur' d'après la proposition 3.

\section{Sur l'existence de séparatrices}

Soit $\omega=0$ de degré de dégénérescence 1 et soient $(x, y)$ des coordonnées (données 
par la proposition 1) telles que

$$
\omega_{\infty}=\mathscr{T}^{\infty} \omega=\frac{1}{2} d\left(\frac{l}{2} y^{2}+\varepsilon x^{l}\right)+\left(\sum_{k \geq m} F_{k} x^{k}\right) d y
$$

où $l \geq 3, m \geq 2, F_{m} \neq 0$. Notons $F \in \mathscr{E}_{2}$ un prolongement de Borel de $\sum F_{k} x^{k}$ $\left(=\mathscr{T}^{\infty} F(x)\right)$ et soit

$$
\Omega=\frac{1}{2} d\left(\frac{l}{2} y^{2}+\varepsilon x^{l}\right)+F(x) d y .
$$

On déduit de la désingularisation des champs de vecteurs ([10], [2] [3], [7]) le lemme suivant:

LEMME 1. Toute séparatrice de $\omega$ est $C^{\infty}$-tangente à une séparatrice de $\Omega$ et inversement.

Ainsi nous supposerons $\omega=\Omega$ pour rechercher les séparatrices.

LEMME 2. Si $2 m<l, \omega=0$ possède une séparatrice.

Démonstration. Soit $\pi:(x, u) \rightarrow\left(x,\left(u-2 F_{m} / l\right) x^{m}\right)$. Après division par $x^{2 m-1}$, l'équation $\pi^{*}(\omega)=0$ s'écrit dans les coordonnées $(x, u), \tilde{\omega}=0$ avec

$$
\mathscr{T}^{-1} \tilde{\omega}=-2 F_{m} u d x .
$$

Elle possède une séparatrice d'équation $x \rightarrow(x, u(x))$, [3]. Son image par $\pi$ est une séparatrice de $\omega=0$.

LEMME 3. Si $2 m \geq l$ et $\varepsilon=-$ ou si $2 m>l, \varepsilon=+$ et l est impair $\omega$ possède une séparatrice.

Démonstration. Le cas $\varepsilon=+, l$ impair se déduit du cas $\varepsilon=-1$ en changeant $x$ en $-x$. Nous supposons $2 m \geq l$ et $\varepsilon=-$. Soit

et soit

$$
\pi:(u, v) \rightarrow\left(u^{2}, v^{l}\right)
$$

$$
\alpha=2 \pi^{*}(\omega)=d\left(\frac{l}{2} v^{2 l}-u^{2 l}\right)+2 l u^{2 m} v^{l-1} f\left(u^{2}\right) d v .
$$

Faisons l'éclatement $E:(u, t) \rightarrow(u, t u)$ de $\alpha$ :

$$
u^{2 l-1} \tilde{\alpha}=E^{*}(\alpha)=u^{2 l-1}\left[\left(-2+l t^{2 l}+2 t^{l} u^{2 m-l} f\left(u^{2}\right)\right) d u+u d t(\cdots)\right] .
$$

Soit $t_{0}$ une racine réelle de

$$
l t^{2 l}-2 \text { si } 2 m>l
$$

ou de

$$
l t^{2 l}+2 F_{m} t^{l}-2 \text { si } 2 m=l .
$$

Le jet d'ordre 1 de $\tilde{\alpha}$ au point $\left(0, t_{0}\right)$ est non dégénéré et son germe possède une séparatrice d'équation $u \rightarrow(u, t(u))$. Son image par $E \circ \pi$ est une séparatrice de $\omega$.

LeMME 4. Si $2 m=l, \varepsilon=1$ et $F_{m}^{2} \geq 2 l$, w possède une séparatrice.

Démonstration. Par les mêmes changements de variables que dans la démonstration du lemme précédent, on obtient

$$
\tilde{\alpha}=\left(2+l t^{2 l}+2 t^{l} f\left(x^{2}\right)\right) d u+u d t(\cdots) .
$$


Le polynome $t^{2 l}+2 t^{l} F_{m}+2$ possède au moins une racine réelle $t_{0}$ et, on montre de la même façon que plus haut que $\omega$ possède une séparatrice.

2. Démonstration de la proposition 2

Comme Lyapunov dans [4] nous allons utiliser les fonctions $c(\theta), s(\theta)$ définies par:

$$
\begin{array}{ll}
\frac{d s}{d \theta}=c(\theta)^{2 n-1}, & s(0)=1, \\
\frac{d c}{d \theta}=-s(\theta), & c(0)=1 .
\end{array}
$$

Elles vérifient les propriétés suivantes:

(i) $n s^{2}(\theta)+c^{2 n}(\theta)=1$,

(ii) $c(\theta)$ et $s(\theta)$ sont périodiques de période

$$
\begin{array}{rlrl}
2 \omega=2 \sqrt{\frac{\pi}{n}} \frac{\Gamma(1 / 2 n)}{\Gamma((n+1) / 2 n)}, & \\
c(\theta+\omega)=-c(\theta), & c(-\theta) & =c(\theta), & c(\omega-\theta)=-c(\theta), \\
s(\theta+\omega)=-s(\theta), & s(-\theta) & =-s(\theta), & s(\omega-\theta)=s(\theta) .
\end{array}
$$

(iii)

$$
\int_{0}^{2 \omega} c^{r}(\theta) d \theta= \begin{cases}0 & \text { si } r \text { impair } \\ c_{r} \neq 0 & \text { si } r \text { pair. }\end{cases}
$$

Dans la suite nous notons $\pi l$ 'application de $\mathbb{R}^{+} \times \mathbb{R}$ dans $\mathbb{R}^{2}$,

$$
\pi:(r, \theta) \rightarrow\left(r c(\theta), r^{n} s(\theta)\right) .
$$

Avec les notations du section précédent on a:

LEMME 5. L'équation $\Omega=\frac{1}{2} d\left(n y^{2}+x^{2 n}\right)+F(x) d y=0$ avec $m>n \geq 2$ ou $m=n \geq 2$ et $F_{m}^{2}<4 m, n$ 'a pas de séparatrice.

Démonstration. Posons $F(x)=x^{m} f(x), \pi^{*}(\Omega)=n r^{2 n-1} \tilde{\Omega}$. On a:

$$
\tilde{\Omega}=\left(1+s(\theta) c(\theta)^{m} r^{m-n} f(r c(\theta)) d r+\frac{r^{m-n+1}}{n} c(\theta)^{m+2 n-1} f(r c(\theta)) d \theta\right.
$$

Si $m>n$, et $r$ est assez petit le coefficient de $d r$ est positif et lorsque $m=n$ et $f^{2}(0)=F_{m}^{2}<4 m$ cette propriété reste vraie car

$$
\left|s(\theta) c(\theta)^{m}\right|<\frac{1}{2 \sqrt{ } m}, \quad \theta \in \mathbb{R} .
$$

Les courbes intégrales de $\tilde{\Omega}=0$ sont les solutions d'une équation différentielle $d r / d \theta=H(r, \theta)$, avec $H>0$ pour $r>0$ assez petit. Leurs images par $\pi$ qui sont les courbes intégrales de $\Omega$ ne sont pas des séparatrices.

Démonstration de la proposition 2. Avec les hypothèses de la proposition, la non existence de séparatrice pour $\omega$ est démontrée par les cinq lemmes précédents. 
Puisque $\omega=\Omega+R$, où $R$ est une 1 -forme dont les coefficients sont plats en $0 \in \mathbb{R}^{2}$, on a:

$$
\frac{1}{n r^{2 n-1}} \pi^{*}(\omega)=\tilde{\omega}=\tilde{\Omega}+\alpha(r, \theta) d r+\beta(r, \theta) d \theta
$$

où $\alpha, \beta$ sont des fonctions $C^{\infty}$ plates en $r$. Comme dans le lemme 5 , la courbe intégrale de $\hat{\omega}$ passant par le point $(\rho, 0)$ s'écrit $r(\rho, \theta)$ avec

$$
\frac{\partial r}{\partial \theta}(\rho, \theta)=K(r(\rho, \theta), \theta) \quad \text { et } r(\rho, 0)=\rho
$$

où $K(r, \theta)$ est $C^{\infty}$, positive pour $r$ assez petit (d'après le lemme 5 et (1)). L'application premier retour $P$ de $\omega=0$ est le germe de $C^{\infty}$-difféomorphisme de $(\mathbb{R}, 0)$

$$
P: \rho \rightarrow r(\rho, 2 \omega) \text {. }
$$

Lorsque $\omega$ est analytique le même type d'argument, que l'on trouve déjà dans Lyapunov [4], montre que $P$ est analytique.

\section{Etude de l'application premier retour}

Dans tout ce section $\omega=0$ désigne une équation sans séparatrice.

Soient $(x, y)$ des coordonnées telles que

$$
\omega_{\infty}=\mathscr{T}^{\infty} \omega=\frac{1}{2} d\left(n y^{2}+x^{2 n}\right)+\left(\sum_{k \geq m} F_{k} x^{k}\right) d y .
$$

De la même façon que dans le section précédent, on note:

$$
\pi(r, \theta)=\left(r c(\theta), r^{n} s(\theta)\right), \quad \pi *(\omega)=n r^{2 n-1} \tilde{\omega} .
$$

Pour alléger les calculs, nous écrirons $r=r(\rho, \theta)$ la solution de $\tilde{\omega}=0$ telle que $r(\rho, 0)=\rho$. Soit

$$
r_{l}=r\left(\bmod \rho^{l+1}\right)=\sum_{k=1}^{l} a_{k}(\theta) \rho^{k}
$$

son développement de Taylor à l'ordre $l$ suivant les puissances de $\rho$. Des expressions (1), (2) de $\tilde{\Omega}$ et $\tilde{\omega}$ du paragraphe précédent on déduit:

$$
\left.\begin{array}{rl}
\left(1+s(\theta) c(\theta)^{m} r_{l}^{m-n} \sum_{k=0}^{l} F_{m+k} r_{l}^{k} c(\theta)^{k}\right) \sum_{k=1}^{l} a_{k}^{\prime}(\theta) \rho^{k} \\
=-\frac{1}{n} r_{l}^{m-n+1} c(\theta)^{m+2 n-1} \sum_{k=0}^{l} F_{m+k} r_{l}^{k} c^{k}(\theta)\left(\bmod \rho^{l+1}\right) .
\end{array}\right\}
$$

Les $a_{k}(\theta)$ pour $k=1,2, \ldots, l$ sont déterminés par $(4)_{l}$.

LEMME 6. Soit $G\left(x^{2}\right)$ un prolongement de Borel de $\sum_{2 k \geq m} F_{2 k} x^{2 k}$. Alors:

$$
\bar{\omega}=\frac{1}{2} d\left(n y^{2}+x^{2 n}\right)+G\left(x^{2}\right) d y
$$

est du type centre. La solution générale $\bar{r}(\rho, \theta)$ de

$$
\frac{\pi^{*}(\bar{\omega})}{n r^{2 n-1}}=\tilde{\bar{\omega}}
$$


est périodique en $\theta$ de période $2 \omega$. En particulier

$$
\bar{r}_{\infty}=\sum_{k \geq 1} \bar{a}_{k}(\theta) \rho^{k} \quad \text { avec } \bar{a}_{k}(\theta)=\bar{a}_{k}(\theta+2 \omega) .
$$

Démonstration. L'équation $\bar{\omega}=0$ est du type centre ou foyer et elle est invariante par l'involution

$$
(x, y) \rightarrow(-x, y) \text {. }
$$

D'après un théorème de Poincaré elle est du type centre et par conséquent $\bar{r}$ est périodique en $\theta$.

LEMME 7. Soit $F_{2 q+1}$ le premier terme d'indice impair dans

Alors

$$
\sum_{k \geq m} F_{k} x^{k}
$$

$$
a_{k}(\theta)=\bar{a}_{k}(\theta) \quad \text { pour } k \leq 2 q+1-n .
$$

Démonstration. Remarquons tout d'abord que (4), s'écrit plus simplement:

$$
\begin{aligned}
& \left(1+s(\theta) c(\theta)^{m} r_{l}^{m-n} \sum_{k=0}^{l-m+n-1} F_{m+k} r_{l}^{k} c(\theta)^{k}\right)\left(\sum_{k=1}^{l} a_{k}^{\prime}(\theta) \rho^{k}\right) \\
& =-\frac{1}{n} r_{l}^{m-n+1} c(\theta)^{m+2 n-1} \sum_{k=0}^{l-m+n-1} F_{m+k} r_{l}^{k} c(\theta)^{k}\left(\bmod \rho^{l+1}\right) .
\end{aligned}
$$

Pour les entiers $l$ tels que:

$$
l+n-1=m+(l-m+n-1) \leq 2 q
$$

les polynômes en $\rho, r_{l}$ et $\bar{r}_{l}$ vérifient la même équation et ainsi:

$$
a_{k}(\theta)=\bar{a}_{k}(\theta) \text { pour } k \leq l \leq 2 q+1-n .
$$

LEMME 8. Si $F_{2 q+1}$ est le premier terme d'indice impair dans

alors

$$
\sum_{k \geq m} F_{k} x^{k}
$$

$$
a_{2 q+2-n}(2 \omega) \neq 0
$$

plus précisément on a

$$
a_{2 q+2-n}(2 \omega)=- \begin{cases}\frac{F_{2 q+1}}{n} \int_{0}^{2 \omega} c(\theta)^{2(q+n)} d \theta, & \text { si } m>n \\ C \neq 0, & \text { si } m=n\end{cases}
$$

où C est une constante dont l'expression sera donnée lors de la démonstration.

Démonstration. Nous allons faire les calculs dans les deux cas:

1er cas: $m>n$. Puisque $m-n>0,(4)_{1}$ s'écrit $\rho a_{1}^{\prime}(\theta)=0$ et

$$
a_{1}(\theta)=a_{1}(0)=1 \text {. }
$$

D'après le lemme 7 , on sait que si $p=2 q+2-n$,

$$
r_{p}=\bar{r}_{p}+b_{p}(\theta) \rho^{p} \quad \text { avec } b_{p}(\theta)=a_{p}(\theta)-\bar{a}_{p}(\theta) .
$$


En reportant cette expression de $r_{p}$ dans $(4)_{p}$ et en tenant compte du fait que $r_{p-1}=\tilde{r}_{p-1}$ est solution de $(4)_{p-1}$, on obtient l'équation suivante:

$$
b_{p}^{\prime}(\theta) \rho^{p}=-\frac{1}{n} c(\theta)^{m+2 n-1} F_{2 q+1} c(\theta)^{2 q+1-m} \rho^{p} .
$$

Puisque par hypothèse $a_{k}(0)=0$ si $k>1$, on déduit du lemme 7

$$
a_{p}(2 \omega)=-\frac{F_{2 q+1}}{n} \int_{0}^{2 \omega} c(\theta)^{2(q+n)} d \theta \neq 0 .
$$

2ème cas: $m=n$ (et $F_{m}^{2}<4 m$ ): Puisque $m=n,(4)_{1}$ s'écrit

$$
\left(1+s(\theta) c(\theta)^{m} F_{m}\right) a_{1}^{\prime}(\theta) \rho=-\frac{1}{n} a_{1}(\theta) c(\theta)^{3 m-1} F_{m} .
$$

(i) $m=2 q+1:\left(1+s(\theta) c(\theta)^{m} F_{m}\right)$ est strictement positif et

$$
\log a_{1}(2 \omega)=-\frac{F_{2 q+1}}{m} \int_{0}^{2 \omega} \frac{c(\theta)^{6 q+2} d \theta}{1+s(\theta) c(\theta)^{m} F_{m}}
$$

est non nul. Ainsi $a_{1}(2 \omega)$ est différent de 1 .

(ii) $m=2 m^{\prime}$ : On obtient

$$
\frac{a_{1}^{\prime}(\theta)}{a_{1}(\theta)}=-\frac{F_{m}}{m} h(\theta)
$$

où

$$
h(\theta)=\frac{c(\theta)^{3 m-1}}{1+s(\theta) c(\theta)^{m} F_{m}} .
$$

Puisque $h(\omega-\theta)=-h(\theta)$, on retrouve le résultat du lemme 7, à savoir:

$$
0=\log a_{1}(2 \omega)=-\frac{F_{2 q+1}}{m}\left(\int_{0}^{\omega} h(\theta) d \theta+\int_{\omega}^{2 \omega} h(\theta) d \theta\right) .
$$

Ecrivons, comme dans le cas $m>n$,

$$
r_{p}=\bar{r}_{p}+b_{p}(\theta) \rho^{p} \quad \text { avec } p=2 q+2-n
$$

et reportons cette expression de $r_{p}$ dans $(4)_{p}$. On obtient:

$$
\begin{aligned}
a_{1}^{\prime}(\theta) & s(\theta) c(\theta)^{m} F_{2 q+1} a_{1}(\theta)^{2 q+1} c(\theta)^{2 q+1}+b_{p}^{\prime}(\theta)\left(1+s(\theta) c(\theta)^{m} F_{m}\right) \\
= & -\frac{1}{n}\left(a_{1}(\theta) F_{2 q+1} c(\theta)^{3 m-1} a_{1}(\theta)^{2 q+1} c(\theta)^{2 q+1}+b_{p}(\theta) c(\theta)^{3 m-1} F_{m}\right) .
\end{aligned}
$$

Après division par

$$
\frac{1}{1+s(\theta) c(\theta)^{m} F_{m}}
$$

cette équation différentielle en $b_{p}(\theta)$ s'écrit:

$$
\begin{aligned}
b_{p}^{\prime}(\theta)-b_{p}(\theta) \frac{a_{1}^{\prime}(\theta)}{a_{1}(\theta)}= & F_{2 q+1} \frac{a_{1}^{\prime}(\theta)}{a_{1}(\theta)} a_{1}(\theta)^{2 q+2} \\
& \times c(\theta)^{2 q+1}\left(s(\theta) c(\theta)^{m}-\frac{1}{F_{m}}\right) .
\end{aligned}
$$


Elle se résout en posant $b_{p}(\theta)=a_{1}(\theta) \lambda(\theta)$ et on a

$$
\begin{aligned}
& \lambda^{\prime}(\theta)=F_{2 q+1} \frac{a_{1}^{\prime}(\theta)}{a_{1}(\theta)}\left(a_{1}(\theta) c(\theta)\right)^{2 q+1}\left(s(\theta) c(\theta)^{m}-\frac{1}{F_{m}}\right) \\
& \lambda^{\prime}(\theta)=-\frac{F_{2 q+1}^{2}}{2 q+1} a_{1}(\theta)^{2 q+1} c(\theta)^{2 q+3 m} \frac{1}{1+s(\theta) c(\theta)^{m} F_{m}}\left(s(\theta) c(\theta)^{m}-\frac{1}{F_{m}}\right) .
\end{aligned}
$$

Les majorations

$$
\left|s(\theta) c(\theta)^{m}\right|<\frac{1}{2 \sqrt{m}} \quad \text { et } \quad F_{m}^{2}<4 m
$$

impliquent que:

$$
1+s(\theta) c(\theta)^{m} F_{m} \text { et } \frac{1}{F_{m}}\left(s(\theta) c(\theta)^{m}-\frac{1}{F_{m}}\right)
$$

sont strictement positifs. Il en est de même pour $a_{1}(\theta)=\exp (\ldots)$. Ainsi $\lambda(2 \omega)$ est non nul et

$$
a_{p}(2 \omega)=\lambda(2 \omega) a_{1}(2 \omega)=\lambda(2 \omega) \neq 0 .
$$

Puisque le jet d'ordre $p=2 q+2-n$ de l'application premier retour $P$ de $\omega$ s'écrit

$$
P_{p}(\rho)=\sum_{k=1}^{p} a_{k}(2 \omega) \rho^{k}
$$

les lemmes $6,7,8$ démontrent la proposition 3 .

Remarque 4. Le point $0 \in \mathbb{R}^{2}$ est un foyer hyperbolique, i.e. $a_{1}(2 \omega) \neq 1$ si et seulement si

$$
p=1=2 q+2-n \geq 2 q+2-m=1,
$$

c'est à dire lorsque $n=m=2 q+1$. Ceci doit pouvoir être interprété en termes de stabilité topologique ou de bifurcation (voir [13]). D'autre part, signalons que, le 'signe de $F_{2 q+1}$ ' permet de décider si 0 est un foyer attractif ou répulsif.

4. $C^{\infty}$-conjugaisons des applications premiers retours et des équations

Soient $\omega=0$ et $\Omega=0$ deux équations de degrés de dégénérescence 1 , sans séparatrice et soient $P$ et $Q$ leurs applications premiers retours. S'il existe un $C^{\infty}$-difféomorphisme $\varphi$ de $\left(\mathbb{R}^{2}, 0\right)$ tel que

$$
\varphi^{*}(\omega) \wedge \Omega=0,
$$

alors $P$ et $Q$ sont $C^{\infty}$-conjuguées par un $C^{\infty}$-difféomorphisme de $(\mathbb{R}, 0)$. Réciproquement on a:

Proposition 4. Soient $\omega=0$ et $\Omega=0\left(\mathscr{T}^{1} \omega=\mathscr{T}^{1} \Omega=y d y\right)$ sans séparatrices qui sont $C^{\infty}$-tangentes, i.e. $\mathscr{T}^{\infty} \omega=\mathscr{T}^{\infty} \Omega$, et telles que leurs applications premiers retours sont $C^{\infty}$-conjuguées. Alors il existe un difféomorphisme $\varphi$ de $\left(\mathbb{R}^{2}, 0\right)$ tel que

$$
\varphi^{*}(\omega) \wedge \Omega=0 \text { et } \mathscr{T}^{\infty} \varphi=\left(1_{\mathbf{R}^{2}}, 0\right) \text {. }
$$

Il est clair que ce résultat du type $C^{\infty}$-conjugaison des applications premiers retours $\Rightarrow C^{\infty}$-conjugaison des équations n'est pas le meilleur possible. Mais il implique évidemment les théorèmes 1 et 2 compte tenu des propositions 1,2 et 3 et du théorème 2 de Takens [11]. 
La démonstration de cette proposition s'appuie sur les mêmes arguments que Takens pour montrer le théorème $3 \mathrm{de}[\mathbf{1 1}]$.

Démonstration. Nous pouvons toujours supposer que:

(i) $\mathscr{T}^{\infty} \omega=\mathscr{T}^{\infty} \Omega=\frac{1}{2} d\left(n y^{2}+x^{2 n}\right)+\sum_{k \geq m} F_{k} x^{k}$;

(ii) les applications premiers retours $P$ et $Q$ de $\omega$ et $\Omega$ évaluées sur le $\frac{1}{2}$-axe des $x \geq 0$ sont identiques.

Soient $\pi: \mathbb{P} \times S^{1} \rightarrow \mathbb{R}^{2}$, où $S^{1}=[0,2 \omega] /\{0=2 \omega\}$,

$$
\begin{gathered}
\pi:(r, \theta) \rightarrow\left(r c(\theta), r^{n} s(\theta)\right) \\
\tilde{\omega}=\frac{\pi^{*}(\theta)}{n r^{2 n-1}}, \quad \tilde{\Omega}=\frac{\pi^{*}(\Omega)}{n r^{2 n-1}} .
\end{gathered}
$$

Il existe deux fonctions $C^{\infty}, A, B: \mathbb{R} \times S^{1} \rightarrow \mathbb{R}$, plate en $r$ le long de $r=0$ telles que

$$
\tilde{\Omega}=\tilde{\omega}+A d r+B r d \theta .
$$

Les germes de feuilletage $F_{\tilde{\Omega}}$ et $F_{\tilde{\omega}}$, définis par $\tilde{\Omega}$ et $\tilde{\omega}$, le long de $\{0\} \times S^{1}$ ont la même holonomie. Ils sont $C^{\infty}$-conjugués par un germe de $C^{\infty}$-difféomorphisme $\phi$ de $\mathbb{R} \times S^{1}$ le long de $0 \times S^{1}$, c'est à dire

$$
\phi^{*}(\tilde{\omega}) \wedge \tilde{\Omega}=0 .
$$

Puisque $\phi$ peut-être construit par relèvement de chemins

$$
c_{\theta}:[0,1] \rightarrow \mathbb{R} \times S^{1}, \quad c_{\theta}(t)=(0, t \theta)
$$

dans les feuilles de $F_{\tilde{\Omega}}$ et $F_{\tilde{\omega}}$, il est clair qu'il peut être choisi de la forme:

$$
\phi:(r, \theta) \rightarrow(r+\psi(r, \theta), \theta)
$$

où $\psi$ est une fonction $C^{\infty}$, plate en $r$ compte tenu de (5).

Soit $\varphi$ l'homéomorphisme $\varphi$ de $\left(\mathbb{R}^{2}, 0\right)$ tel que

$$
\varphi \circ \pi=\pi \circ \phi \text {. }
$$

Puisque la restriction de $\pi$ à $] 0, \infty) \times S^{1}$ est un $C^{\infty}$-difféomorphisme sur $\mathbb{R}^{2}-\{0\}$, $\varphi$ s'écrit

$$
\varphi(x, y)=\left(x+\varphi_{1}(x, y), y+\varphi_{2}(x, y)\right)
$$

où $\varphi_{1}, \varphi_{2}$ sont des fonctions $C^{\infty}$ en dehors de 0 qui sont déterminées par

$$
\begin{aligned}
& \varphi_{1}\left(r c(\theta), r^{n} s(\theta)\right)=c(\theta) \psi(r, \theta), \\
& \varphi_{2}\left(r c(\theta), r^{n} s(\theta)\right)=s(\theta)\left[(r+\psi(r, \theta))^{n}-r^{n}\right]=s(\theta) \psi_{1}(r, \theta),
\end{aligned}
$$

où $\psi_{1}$ est encore une fonction plate en $r$. La proposition 4 est alors une conséquence du lemme suivant:

LEMME 9. Soit $f:\left(\mathbb{R}^{2}, 0\right) \rightarrow(\mathbb{R}, 0)$ telle que $F$ définie par

$$
F(r, \theta)=f\left(r c(\theta), r^{n} s(\theta)\right)
$$

soit $C^{\infty}$, plate en r le long de $r=0$. Alors $f$ est $C^{\infty}$, plate en $0 \in \mathbb{R}^{2}$.

Démonstration. Le même argument que plus haut montre que $f$ est continue, $C^{\infty}$ en dehors de $0 \in \mathbb{R}^{2}$. Il suffit donc de montrer que pour tout $(i, j) \in \mathbb{N}^{2}$ les

$$
\frac{\partial^{i+j} f}{\partial x^{i} \partial y^{j}}(x, y)
$$


se prolongent en des fonctions continues, nulles en $0 \in \mathbb{R}^{2}$. En fait, il suffit de montrer que

$$
\frac{\partial f}{\partial x}\left(r c(\theta), r^{n} s(\theta)\right) \text { et } \frac{\partial f}{\partial y}\left(r c(\theta), r^{n} s(\theta)\right)
$$

sont encore plates en $r$, le cas général s'en déduisant par induction. Un calcul élémentaire montre que:

$$
\begin{gathered}
d r=c(\theta)^{2 n-1} d x+\frac{s(\theta)}{r^{n-1}} d y, \quad d \theta=-n \frac{s(\theta)}{r} d x+\frac{c(\theta)}{r^{n}} d y \\
\frac{\partial f}{\partial x}\left(r c(\theta), r^{n} s(\theta)\right)=\frac{\partial F}{\partial r}(r, \theta) c(\theta)^{2 n-1}+\frac{\partial F}{\partial \theta}(r, \theta)\left(-\frac{n s(\theta)}{r}\right) \\
\frac{\partial f}{\partial y}\left(r c(\theta), r^{n} s(\theta)\right)=\frac{\partial F}{\partial r}(r, \theta) \frac{s(\theta)}{r^{n-1}}+\frac{\partial F}{\partial \theta}(r, \theta) \frac{c(\theta)}{r^{n}}
\end{gathered}
$$

et ces deux dérivées partielles sont plates en $r$.

\section{REFERENCES}

[1] I. Bendixson. Sur les points singuliers des équations différentielles. Ofv. Kangl. Vetenskaps. Akade. Eörhandlinger. Stokholm. 9 (1898), 635-658.

[2] F. Dumortier, P. Rodriguez \& R. Roussarie. Germs of diffeomorphisms in the plane. Lecture Notes in Math. No. 902. Springer: Berlin, 1975.

[3] F. Dumortier. Singularities of vector fields on a plane. J. Diff. Equns. 23 (1977), 53-106.

[4] A. M. Lyapunov. Stability of Motion, pp. 123-194. Academic Press: New York, 1966.

[5] R. Moussu: Sur un théorème de Poincaré-Lyapunov. Astérisque. (To appear.)

[6] R. Moussu. Sur l'existence d'intégrales premières pour un germe de forme de Pfaff. Ann. Inst. Fourier 26 (1976) 229-237.

[7] J. F. Mattei \& R. Moussu. Holonomie et intégrales premières. Ann. Scient. Ec. Norm. Sup. Ser. 4. $13(1980), 469-523$.

[8] H. Poincaré. Mémoire sur les courbes définies par une équation differentielle. J. Math. Pures et Appl. 3-7 (1881), 375-422.

[9] R. Roussarie. Modèles locaux de champs et formes. Astérisque 30 (1975).

[10] A. Seidenberg. Reduction of singularities of the differential equation $A d y=B d x$. Amer. J. Math. 79 (1968), 248-269.

[11] F. Takens. Normal forms for certain singularities of vector fields. Ann. Inst. Fourier-Grenoble. 23 (1973) 163-195.

[12] F. Takens. Singularities of vector fields. Publ. Math. I.H.E.S. 43 (1974), 47-100.

[13] F. Takens. Forced oscillations and bifurcations. Comm. Math. Inst. Rijkcuniversiteit Utrecht 3 (1974), 1-59. 